\title{
Inhibidores de la bomba de protones por vía intravenosa en la hemorragia por úlcera péptica: ¿es necesaria la supresión ácida máxima para disminuir el resangrado?
}

\author{
A. Garrido, A. Giráldez, C. Trigo, E. Leo, A. Guil y J. L. Márquez \\ Servicio de Aparato Digestivo. Hospital Virgen del Rocío. Sevilla
}

\section{RESUMEN}

Objetivo: comparar dos pautas de pantoprazol por vía intravenosa en pacientes con hemorragia digestiva alta (HDA) ulcerosa de alto riesgo para presentar persistencia o recidiva hemorrágica.

Material y método: se randomizaron los pacientes en dos grupos: grupo 0: tratamiento con bolo de $80 \mathrm{mg}$ i.v. de pantoprazol y perfusión continua a $8 \mathrm{mg} / \mathrm{h}$ durante 72 horas; grupo 1: tratamiento con $40 \mathrm{mg}$ i.v. de pantoprazol diarios. Se analizó el porcentaje de persistencia/recidiva hemorrágica entre ambos grupos, requerimientos transfusionales, necesidad de cirugía y mortalidad del episodio hemorrágico.

Resultados: se incluyeron 20 pacientes en el grupo 0 y 21 en el grupo 1 . No se encontraron diferencias entre ambos grupos en cuanto al sexo, edad, hábito tabáquico, consumo de AINE, presencia de inestabilidad hemodinámica, estigma sobre el nicho ulceroso (Forrest la 5 vs. 14,3\%, p = 0,322; Forrest Ib 30 vs. $33,3 \%, p=0,819$; Forrest IIa 60 vs. $50,1 \%, p=0,753$ ). El $90 \%$ de los pacientes del grupo 0 recibió tratamiento endoscópico vs. el $100 \%$ del grupo $1, p=0,232$. El $50 \%$ de los pacientes del grupo 0 recibió transfusión $v$ s. el $52,4 \%$ del grupo $1, p=0,879$. Dos pacientes $(10,5 \%)$ del grupo 0 presentaron recidiva hemorrágica us. 3 pacientes (14,3\%) del grupo 1, precisando cirugía 1 paciente de cada grupo y falleciendo 1 paciente del grupo 0 .

Conclusiones: la inhibición ácida máxima de la secreción ácida gástrica mediante bolo e infusión continua de pantoprazol no ofrece resultados superiores al tratamiento con dosis convencionales en el episodio hemorrágico agudo.

Palabras clave: Hemorragia digestiva. Inhibidores de la bomba de protones. Pantoprazol. Ullcera péptica.

\begin{abstract}
Objective: to compare two regimens of pantoprazole administered intravenously in patients with ulcerative gastrointestinal bleeding (UGB), and a high risk of presenting with persitent or recurrent hemorrhage.

Material and method: patients were randomized into two groups: group 0 - treatment with a $80 \mathrm{mg}$ bolus of pantoprazole administered intravenously, followed by continuous infusion of 8 $\mathrm{mg} / \mathrm{h}$ for 72 hours; group 1 - treatment with $40 \mathrm{mg}$ of pantoprazole administered intravenously on a daily basis. The percentage of hemorrhagic persistence/recurrence in both groups was analyzed, as were transfusion requirements, need for surgery, and mortality resulting from the hemorrhagic episode.

Results: there were 20 patients in group 0 and 21 in group 1. No differences were found between groups in terms of gender, age, smoking habits, use of NSAIDs, presence of hemodynamic instability or stigmata in ulcer crater (Forrest la: 5 vs. $14.3 \%, p=$ 0.322; Forrest Ib: 30 vs. 33.3\%, $p=0.819$; Forrest Ila: 60 vs. $50.1 \%, p=0.753)$. In group $0,90 \%$ of patients received endoscopic treatment, versus $100 \%$ in group $1, p=0.232$. In group $0,50 \%$ of patients had a transfusion, as compared to $52.4 \%$ in group $1, \mathrm{p}=0.879$. In group 0,2 patients $(10.5 \%)$ presented with recurrent hemorrhage, versus 3 patients $(14.3 \%)$ in group 1 . Surgery was required by 1 person from each group, and 1 patient in group 0 died.

Conclusions: maximum acid inhibition with a bolus and a then a continuous infusion of pantoprazole does not yield better results than treatment with conventional doses in acute hemorrhagic episodes.
\end{abstract}

Key words: Digestive haemorrhage. Proton pump inhibitors. Pantoprazole. Peptic ulcer.

Garrido A, Giráldez A, Trigo C, Leo E, Guil A, Márquez JL. Inhibidores de la bomba de protones por vía intravenosa en la hemorragia por úlcera péptica: ¿es necesaria la supresión ácida máxima para disminuir el resangrado? Rev Esp Enferm Dig 2008; 100: 466-469.

Recibido: 15-04-08.

Aceptado: 06-05-08.

Correspondencia: Antonio Garrido Serrano. C/ Carmen de Burgos, 19. 41940 Tomares, Sevilla.e-mail: agarser@telefonica.net

\section{INTRODUCCIÓN}

La hemorragia gastrointestinal por úlcera péptica continúa siendo una de las urgencias digestivas más frecuen- 
tes y el tratamiento con fármacos inhibidores de la bomba de protones (IBP) constituye la terapia médica de elección (1), ya que disminuye el riesgo de recidiva hemorrágica debido a la potente inhibición de la secreción ácida que produce su administración (2).

Sin embargo, a diferencia de los resultados claros y consistentes obtenidos en los ensayos clínicos demostrando la eficacia de la terapia endoscópica en el tratamiento de la HDA por úlcera (3), la eficacia clínica de los IBP ofrece todavía cierta controversia y los datos disponibles actualmente no son definitivos en cuanto a la forma de administración y dosis óptima para este tipo de pacientes (4), incluso habiéndose descrito diferencias en cuanto a los resultados dependiendo de la localización geográfica (5).

En este trabajo nos proponemos estudiar si la inhibición ácida profunda mediante bolo e infusión continua de pantoprazol es más eficaz que dosis convencionales en pacientes con HDA ulcerosa con estigmas endoscópicos de riesgo de resangrado.

\section{MATERIAL Y MÉTODOS}

Se ha realizado un estudio prospectivo y randomizado de pacientes consecutivos ingresados en la Unidad de Sangrantes del Hospital Virgen del Rocío de Sevilla por HDA ulcerosa. Se recogieron los siguientes datos de todos los pacientes: edad, sexo, hábito tabáquico, consumo de AINE, patología de base (6) (enfermedades que aumentan la comorbilidad del episodio hemorrágico), presencia de inestabilidad hemodinámica (cuando se presentaban dos o más de los siguientes criterios: presión arterial sistólica inferior a $100 \mathrm{mmHg}$, frecuencia cardiaca superior a $100 \mathrm{spm}$, presencia de signos de hipoperfusión periférica, cambios en la tensión arterial o frecuencia cardiaca tras el ortostatismo), estigma del nicho ulceroso según la clasificación de Forrest y el tratamiento endoscópico aplicado en los casos de hemorragia activa, vaso visible y coágulo inestable. Los enfermos eran asignados desde el momento de ingreso en la unidad a dos grupos de manera randomizada: el grupo 0 que recibía bolo de $80 \mathrm{mg}$ i.v. de pantoprazol y perfusión continua a $8 \mathrm{mg} / \mathrm{h}$ durante 72 horas, lo que consigue una supresión ácida profunda; y el grupo 1, que recibía $40 \mathrm{mg}$ i.v. de pantoprazol durante 72 horas. Entre las primeras 24-48 horas de ingreso se realizaba un nuevo control endoscópico con retratamiento si persistían estigmas de riesgo de hemorragia. Se analizó el porcentaje de persistencia/recidiva hemorrágica entre ambos grupos, requerimientos transfusionales, necesidad de cirugía y mortalidad del episodio hemorrágico. Los pacientes dieron su consentimiento informado por escrito previo a la inclusión en el estudio.

\section{Estadística}

Se incluyeron diversas variables cuantitativas y cualitativas, expresándolas como media o porcentaje respecti- vamente. Se empleó el test de la t de Student para la comparación de variables cuantitativas y de la Chi cuadrado para variables cualitativas. Se realizó un análisis de regresión logística multivariante para investigar los factores asociados a persistencia/recidiva hemorrágica.

Los valores de $\mathrm{p}<0,05$ se consideraron estadísticamente significativos.

\section{RESULTADOS}

En la tabla I se recogen las características basales de los pacientes, comprobando que ambos grupos de tratamiento son homogéneos respecto a edad, sexo, patología de base, hábito tabáquico, consumo de AINE, presencia de inestabilidad hemodinámica y grado de Forrest en la endoscopia inicial al ingreso. Se aplicó terapéutica endoscópica al $90 \%$ de los pacientes del grupo 0 frente al $100 \%$ de los pacientes del grupo $1(\mathrm{p}=0,232)$, consistiendo en todos los casos en la inyección de adrenalina diluida $1 / 10.000$ y polidocanol al $2 \%$; ningún paciente precisó una segunda terapia en el control endoscópico precoz, pues todos presentaban úlceras de fondo fibrinoso limpio o con manchas planas de hematina. En cuanto a la evolución de los pacientes, en la tabla II se muestra que no hubo diferencias entre ambos grupos en cuanto a necesidades transfusionales, recidiva hemorrágica, tratamiento quirúrgico o mortalidad.

El estudio de regresión logística multivariante no encontró ningún factor de riesgo asociado a recidiva hemorrágica, incluido la diferente modalidad de tratamiento con pantoprazol utilizada.

Tabla I. Características generales de la muestra $(n=41)$

\begin{tabular}{lccc}
\hline & Grupo 0 $(n=20)$ & Grupo 1 $(n=21)$ & $p$ \\
\hline Edad (años) & $60,05 \pm 16,75$ & $60,67 \pm 13,34$ & $0,892^{*}$ \\
Sexo (\% hombres) & 80 & 85 & $0,471^{* *}$ \\
Hábito tabáquico (\%) & 55 & 45 & $0,654^{* *}$ \\
Consumo de AlNE (\%) & 35 & 65 & $0,085^{* *}$ \\
Inestabilidad & & & \\
$\quad$ hemodinámica (\%) & 50 & 76,2 & $0,082^{* *}$ \\
Patología de base (\%) & 42,1 & 47,6 & $0,726^{* *}$ \\
Forrest la (\%) & 5 & 14,3 & $0,322^{* *}$ \\
Forrest Ib (\%) & 30 & 33,3 & $0,819^{* *}$ \\
Forrest Ila (\%) & 60 & 50,1 & $0,753^{* *}$ \\
\hline
\end{tabular}

${ }^{*}$ de Student. ${ }^{*}$ Chi cuadrado.

Tabla II. Evolución de los pacientes de la muestra $(n=41)$

\begin{tabular}{lccc}
\hline & Grupo $0(n=20)$ & Grupo $1(n=21)$ & $p$ \\
\hline Necesidades transfusión (\%) & 50 & 52,4 & $0,879^{*}$ \\
Recidiva hemorrágica $(n)$ & 2 & 3 & $0,549^{*}$ \\
Necesidad de cirugía $(n)$ & 1 & 1 & $0,731^{*}$ \\
Mortalidad $(n)$ & 1 & 0 & $0,475^{*}$ \\
\hline
\end{tabular}

*Chi cuadrado. 


\section{DISCUSIÓN}

El tratamiento médico con IBP sin aplicación de hemostasia endoscópica no se ha mostrado eficaz para detener el sangrado ni prevenir la recidiva en la HDA por ulcus péptico (7), en cambio el advenimiento del tratamiento endoscópico en la HDA ulcerosa es, sin duda, el avance más importante en el manejo de estos pacientes, muy superior respecto al tratamiento farmacológico; no obstante, estudios realizados in vitro han demostrado que el control mantenido del pH intragástrico por encima de 6 debería acompañarse de una mejora de los mecanismos de hemostasia de la úlcera sangrante (8), así la administración i.v. continuada de omeprazol y pantoprazol consigue un $\mathrm{pH}$ superior a 6 durante más del $90 \%$ del tiempo que se está administrando (9), lo cual se ha demostrado igualmente con la administración por vía oral de dichos fármacos en un trabajo reciente (10).

Sin embargo, aunque los IBP han sido los fármacos que han demostrado mayor eficacia en el manejo de estos enfermos por su mayor potencia para la inhibición ácida, los metaanálisis y revisiones sistemáticas más importantes han arrojado resultados que no aclaran el esquema terapéutico ideal. Así, en un estudio se aleatorizan pacientes con HDA ulcerosa que han sido sometidos a tratamiento endoscópico a recibir placebo u omeprazol en bolo de $80 \mathrm{mg}$ seguido de perfusión i.v. de $8 \mathrm{mg} / \mathrm{hora}$ durante 3 días; el grupo tratado con omeprazol presentó una tasa de recidiva de la hemorragia del $4,2 \%$ frente a $20 \%$ del grupo placebo (11); en cambio, en una revisión sistemática más reciente que incluye 24 ensayos randomizados y controlados con un total de 4.373 pacientes para evaluar la eficacia de los IBP en la hemorragia ulcerosa, se concluye que no hubo diferencias en la mortalidad entre el grupo control y el tratado con IBP, pero los IBP comparados con placebo sí disminuyeron significativamente las tasas de recidiva hemorrágica y necesidades de cirugía, aunque las necesidades quirúrgicas fueron similares al compararlos con fármacos anti- $\mathrm{H}_{2}$. Además, se demuestra que ni la vía de administración del IBP ni los diferentes esquemas posológicos utilizados influyeron en los resultados (12). Siguiendo una metodología similar, nuevamente se confirma que no existe evidencia de que el tratamiento con IBP afecte resultados clínicamente importantes tales como la mortalidad, resangrado o necesidad de cirugía, aunque se demuestra que su empleo previo a la realización de la endoscopia en pacientes con HDA ulcerosa reduce significativamente la proporción de pacientes con estigmas endoscópicos de sangrado reciente y riesgo de hemorragia (13).

Nuestro hospital cuenta con un médico adjunto de digestivo de guardia de presencia física, por lo que a los pacientes que acuden por HDA se les realiza endoscopia urgente (menos de 6 horas) en todos los casos, aplicando tratamiento endoscópico cuando está indicado; además, para este estudio a los enfermos se les revisaba sistemáticamente el estado de la lesión transcurridas entre 24-48 ho- ras, lo que se ha venido en llamar el second-look endoscópico, cuyo objetivo es detectar de forma precoz lesiones que puedan sufrir una recidiva hemorrágica y aplicar un segundo tratamiento endoscópico, lo cual ha demostrado que reduce el riesgo de recidiva de la hemorragia (14). Pues bien, aplicando esta sistemática no hemos encontrado diferencias en los resultados entre los pacientes que han recibido un tratamiento con IBP para conseguir una inhibición máxima del ácido frente al tratamiento con dosis convencionales, con el consiguiente ahorro que ello supone. Este hallazgo, a pesar de los estudios in vitro comentados previamente, puede explicarse en base a que el calibre de la arteria que ocasiona una hemorragia de alto riesgo oscila entre 1,5 y $3,4 \mathrm{~mm}$, frente a los $0,1-1,8 \mathrm{~mm}$ en caso de hemorragias leves y limitadas, lo cual puede explicar la necesidad de algún método físico, más importante que el farmacológico del control del ácido, para conseguir el cese de la hemorragia y prevenir su recidiva causada por este tipo de lesiones (15).

Así pues, el manejo del enfermo sangrante por úlcera gastroduodenal deberá individualizarse a cada medio y centro hospitalario concreto, pero en aquellos donde se pueda aplicar una terapéutica endoscópica eficaz y precoz (16), el tratamiento con IBP a dosis máximas no parece superior al tratamiento con dosis convencionales.

$\mathrm{Al}$ margen de los resultados de este trabajo, se ha publicado recientemente una alta frecuencia de uso incorrecto de los IBP, siendo recomendable una utilización más racional de los mismos para evitar posibles efectos secundarios, interacciones con otros fármacos y aportar una atención médica más eficiente (17).

\section{BIBLIOGRAFÍA}

1. Khuroo MS, Farahat KL, Kagevi IE. Treatment with proton pump inhibitors in acute non-variceal upper gastrointestinal bleeding: a metaanalysis. J Gastroenterol Hepatol 2005; 20: 11-25.

2. Olsen KM. Use of acid suppression therapy for treatment of non-variceal upper gastrointestinal bleeding. Am J Health Syst Pharm 2005; 62: 18-23.

3. Cook DJ, Guyatt GH, Salena BJ, Laine LA. Endoscopic therapy for acute nonvariceal upper gastrointestinal hemorrhage: a meta-analysis. Gastroenterology 1992; 102: 139-48.

4. Armstrong D. Intravenous proton pump inhibitor therapy: a rationale for use. Rev Gastroenterol Disord 2005; 2: 18-30.

5. Leontiadis GI, Sharma VK, Howden CW. Systematic review and meta-analysis: enhanced efficacy of proton pump inhibitor therapy for peptic ulcer bleeding in Asia - a post hoc analysis from the Cochrane Collaboration. Aliment Pharmacol Ther 2005; 21: 1055-61.

6. Garrido A, Márquez JL, Guerrero FJ, Leo E, Pizarro MA. Cambios en la etiología, resultados y características de los pacientes con hemorragia digestiva aguda grave a lo largo del periodo 1999-2005. Rev Esp Enferm Dig 2007; 99: 275-9.

7. Julapalli VR, Graham DY. Appropriate use of intravenous proton pump inhibitors in the management of bleeding peptic ulcer. Dig Dis Sci 2005; 50: 1185-93.

8. Patchett SE, Euright H, Afdahl N, O'Connell W, O'Donoghue DP. Clot lysis by gastric juice: an in vitro study. Gut 1989; 30: 1704-7.

9. Van Rensburg CJ, Hatmann M, Thorpe A, Venter L, Theron I, Luhmann $\mathrm{R}$, et al. Intragastric $\mathrm{pH}$ during continuous infusion with pantoprazole in patients with bleeding peptic ulcer. Am J Gastroenterol 2003; 98: 2635-41. 
10. Laine L, Shah A, Bemanian S. Intragastric ph with oral vs. intravenous bolus plus infusion proton pump inhibitor therapy in patients with bleeding ulcers. Gastroenterology 2008; 3: 301-7.

11. Lau JY, Sung JJ, Lee KK, Yung MY, Wong SK, Wu JC, et al. Effect of intravenous omeprazole on recurrent bleeding after endoscopic treatment of bleeding peptic ulcers. N Engl J Med 2000; 343: 310-6.

12. Leontiadis GI, Sharma VK, Howden CW. Proton pump inhibitor treatment for acute peptic ulcer bleeding. Cochrane Database Syst Rev 2004; (3): CD002094.

13. Dorward S, Sreedharan A, Leontiadis GI, Howden CW, Moayyedi P, Forman D. Proton pump inhibitor treatment initiated prior to endoscopic diagnosis in upper gastrointestinal bleeding. Cochrane Database Syst Rev 2006; (4): CD005415.

14. Marmo R, Rotondano G, Bianco MA, Piscopo R, Prisco A, Cipolleta
L. Outcome of endoscopic treatment for peptic ulcer bleeding: is a second look necessary? A meta-analysis. Gastroint Endosc 2003; 98 : 86-97.

15. Swain CP, Storey DW, Bown SG. Nature of the bleeding vessel in recurrently bleeding gastric ulcers. Gastroenterology 1986; 90: 595608.

16. Garrido Serrano A, Guerrero Igea FJ, Perianes Hernández C, Arenas Posadas FJ, Palomo Gil S. Inyección local terapéutica en el ulcus péptico sangrante: estudio comparativo de adrenalina frente a adrenalina más agente esclerosante. Rev Esp Enferm Dig 2002; 94: 401-5.

17. Martín-Echevarría E, Pereira A, Torralba M, Arriola G, Martín P, Mateos J, et al. Evaluación del uso de los inhibidores de la bomba de protones en un servicio de medicina interna. Rev Esp Enferm Dig 2008; 100: 76-81. 